\title{
Some groups of finite homological type
}

\author{
Ian J. Leary* Müge Saadetoğlu ${ }^{\dagger}$
}

November 2, 2005

\begin{abstract}
For each $n \geq 0$ we construct a torsion-free group that satisfies K. S. Brown's FHT condition and is $F_{n}$ (and hence $F P_{n}$ ), but is not $F P_{n+1}$.
\end{abstract}

\section{Introduction}

While working on comparing different notions of Euler characteristic, K. S. Brown introduced a new homological finiteness condition for discrete groups [6, IX.6]. The group $G$ is said to be of finite homological type or $F H T$ if $G$ has finite virtual cohomological dimension, and for every $G$-module $M$ whose underlying abelian group is finitely generated, the homology groups $H_{i}(G ; M)$ are all finitely generated. If $G$ is $F H T$, then one may define a 'naïve Euler characteristic' for every finite-index subgroup $H$ of $G$, as the alternating sum of the dimensions of the homology groups of $H$ with rational coefficients.

One question that arises is the connection between FHT and the usual homological finiteness conditions $F P$ and $F P_{n}$, which were introduced by J.-P. Serre [9], and the topological finiteness conditions $F$ and $F_{n}$, which we believe were first studied by C. T. C. Wall. (We shall define these conditions below.) It is easy to see that any group $G$ of type $F P$ is $F H T$, and one might conjecture that every torsion-free group that is $F H T$ is also of type $F P$. The aim of this paper is to show that this is not the case. For each

\footnotetext{
*Partially supported by NSF grant DMS-0505471

${ }^{\dagger}$ Supported by the British Council and by the Ohio State Mathematical Research Institute
} 
$n \geq 0$, we exhibit a torsion-free group $G_{n}$ that is $F H T$ and of type $F_{n}$, but that is not of type $F P_{n+1}$. (Note that type $F_{n}$ implies type $F P_{n}$.)

Our construction is based on R. Bieri's construction of a group that is $F P_{n}$ but not $F P_{n+1}[3$, Prop 2.14]. We also use G. Higman's group $H$ which has the properties that it has no non-trivial finite quotients and that it is acyclic (i.e., has the same integral homology as the trivial group) $[1,7]$. The group $G_{0}$ is just an infinite free product of copies of $H$, and for $n>0, G_{n}$ may be described as a free product of two groups of type $F$, amalgamating a common subgroup isomorphic to $G_{n-1}$.

A construction of the groups $G_{n}$ was given in the University of Southampton $\mathrm{PhD}$ thesis of the second named author [8].

\section{Definitions}

Let $G$ be a discrete group, let $\mathbb{Z} G$ be the integral group ring of $G$, and let $\mathbb{Z}$ stand for the trivial $\mathbb{Z} G$-module, i.e., the module whose underlying abelian group is infinite cyclic upon which each element of $G$ acts as the identity. Modules will be left modules unless otherwise stated.

We begin by recalling some classical homological finiteness conditions, which were introduced by J.-P. Serre [9], but see also [3, Ch I] and [6, Ch VIII]. A projective resolution $P_{*}$ for $\mathbb{Z}$ over $\mathbb{Z} G$ is an exact sequence

$$
\cdots \rightarrow P_{i} \rightarrow \cdots \rightarrow P_{1} \rightarrow P_{0} \rightarrow \mathbb{Z} \rightarrow 0
$$

of $\mathbb{Z} G$-modules in which each $P_{i}$ is projective. The group $G$ is $F P_{n}$ if there exists $P_{*}$ such that $P_{i}$ is finitely generated for all $i \leq n$ and is $F P_{\infty}$ if there is a $P_{*}$ in which $P_{i}$ is finitely generated for all $i$. The group $G$ is of finite cohomological dimension if there exists $P_{*}$ in which some $P_{n}=0$, in which case we may take $P_{i}=0$ for all $i \geq n$. It can be shown that any group of finite cohomological dimension is torsion-free. The group $G$ is $F P$ if $G$ is of finite cohomological dimension and of type $F P_{\infty}$. If $G$ is $F P$, then there exists a resolution $P_{*}$ in which each $P_{i}$ is finitely generated and only finitely many $P_{i}$ are non-zero.

The condtions $F P$ and $F P_{n}$ are motivated by topology. The group $G$ is of type $F$ if there is a model for the classifying space $B G$ that has only finitely many cells. The group $G$ is type $F_{n}$ if there is a model for $B G$ that has only finitely many cells of dimension less than or equal to $n$. The cellular chain complex of the universal cover of a model for $B G$ is a resolution for $\mathbb{Z}$ by 
free $\mathbb{Z} G$-modules. In particular, if $G$ is type $F$, then $G$ is type $F P$, and if $G$ is type $F_{n}$ then $G$ is type $F P_{n}$. The following three conditions on a group $G$ are equivalent: $G$ can be finitely generated; $G$ is $F P_{1} ; G$ is type $F_{1}$. A group $G$ is type $F_{2}$ if and only if $G$ can be finitely presented. There exist groups of type $F P_{2}$, and even groups of type $F P$, that cannot be finitely presented [2]. For any $n \geq 2$, the following two conditions on a group $G$ are equivalent: $G$ is type $F_{n} ; G$ can be finitely presented and is $F P_{n}$ [6, Exercise VIII.7.2].

If $G$ contains a finite-index subgroup $H$ which is of finite cohomological dimension, $G$ is said to be of finite virtual cohomological dimension or finite ved. By an argument due to Serre, any group of finite vcd admits an action with finite stabilizers on a finite-dimensional contractible CW-complex [6, Theorem VIII.11.1].

Remark 1 Since there are many interesting discrete groups that are not virtually torsion-free, one might argue that the condition 'finite ved' is an unnatural one, which should be replaced by the condition 'admits an action with finite stabilizers on a finite-dimensional contractible space' whenever possible.

Brown defines a group $G$ to be $F H T$ if $G$ is of finite vcd, and for every right $G$-module $M$ whose underlying abelian group is finitely-generated, each of the homology groups $H_{i}(G, M)$ is a finitely generated abelian group [6, IX.6]. These homology groups may be computed as the homology of the chain complex

$$
M \otimes_{\mathbb{Z} G} P_{*}
$$

for any projective resolution $P_{*}$ for $\mathbb{Z}$ over $\mathbb{Z} G$. The homology groups of a chain complex of finitely generated abelian groups are themselves finitely generated. Hence each $H_{i}(G, M)$ is finitely generated whenever $G$ is type $F P_{\infty}$, and we see that any group $G$ of finite ved that is of type $F P_{\infty}$ is $F H T$. In particular, any torsion-free group of type $F P$ is $F H T$.

Remark 2 In his original papers on Euler characteristics, Brown gave a different definition of finite homological type, which we shall call $F H T^{\prime}$. The group $G$ is is $F H T^{\prime}$ if $G$ is of finite vcd, and for each torsion-free finiteindex subgroup $H \leq G$, the integral homology groups $H_{i}(H ; \mathbb{Z})$ are finitely generated $[4,5]$. By Shapiro's lemma, $H_{i}(H ; \mathbb{Z}) \cong H_{i}(G ; \mathbb{Z}[H \backslash G])$, where $\mathbb{Z}[H \backslash G]$ denotes the permutation module with basis the right cosets of $H$ in $G$. Hence any group that is $F H T$ is also $F H T^{\prime}$. We do not know whether $F H T$ is equivalent to $F H T^{\prime}$. 


\section{Prerequisites}

Here we collect together some known results that are used in our construction.

Proposition 3 Let $A$ be a finitely generated abelian group. There exists $n$ such that $G=\operatorname{Aut}(A)$ is isomorphic to a subgroup of $G L_{n}(\mathbb{Z})$.

Proof. Let $T(A)$ be the torsion subgroup of $A$, and let $A^{\prime}$ be a complement to $T(A)$, so that $A=T(A) \oplus A^{\prime}$ and $A^{\prime} \cong \mathbb{Z}^{r}$ for some $r \geq 0$. The subgroup $T(A)$ is characteristic in $A$ and so there is a natural surjection

$$
\phi: G \rightarrow \operatorname{Aut}(T(A)) \oplus \operatorname{Aut}(A / T(A)) .
$$

Let $H$ be the subgroup of $G$ consisting of those $f \in G$ such that $f\left(A^{\prime}\right)=A^{\prime}$. Then $H$ is a direct product

$$
H=\operatorname{Aut}(T(A)) \oplus \operatorname{Aut}\left(A^{\prime}\right) \cong \operatorname{Aut}(T(A)) \oplus G L_{r}(\mathbb{Z}),
$$

and $\phi$ induces an isomorphism from $H$ to $\operatorname{Aut}(T(A)) \oplus \operatorname{Aut}(A / T(A))$. Elements $f \in \operatorname{ker}(\phi)$ act as the identity on $T(A)$, and for each $a \in A^{\prime}$, $f(a)=a+b$ for some $b \in T(A)$. It follows that $\operatorname{ker}(\phi)$ is isomorphic to $\operatorname{Hom}\left(A^{\prime}, T(A)\right) \cong T(A)^{r}$, and so $\operatorname{ker}(\phi)$ is a finite group. Since $\phi$ restricted to $H$ is an isomorphism, it follows that the index of $H$ in $G$ is equal to the order of $\operatorname{ker}(\phi)$, and so the index of $H$ in $G$ is finite.

The finite group $\operatorname{Aut}(T(A))$ is isomorphic to a subgroup of $G L_{s}(\mathbb{Z})$ for some $s$ (for example, $s=|\operatorname{Aut}(T(A))|$ will suffice). Hence $H$ is isomorphic to a subgroup of $G L_{r+s}(\mathbb{Z})$. Equivalently, there is a faithful $H$-module $N$ whose underlying abelian group is free abelian of rank $r+s$. If the index of $H$ in $G$ is $m$, then the induced module $\mathbb{Z} G \otimes_{\mathbb{Z} H} N$ is a faithful $G$-module whose underlying abelian group is free abelian of rank $n=m(r+s)$. The action map for this module is an embedding of $G$ in $G L_{n}(\mathbb{Z})$.

Lemma 4 Let $X$ be a connected $C W$-complex, let $Y$ be a connected subcomplex, and let $y_{0} \in Y$ be a basepoint for both spaces. Let $G$ be $\pi_{1}\left(X, y_{0}\right)$, the fundamental group of $X$, let $i: \pi_{1}\left(Y, y_{0}\right) \rightarrow G$ be the induced map of fundamental groups, and let $H$ be a subgroup of $G$. Let $\widehat{X}$ be the covering space of $X$ with fundamental group $H$ and let $\widehat{Y}$ be the subspace of $\widehat{X}$ consisting of lifts of points of $Y$. There is a bijective correspondence between components of $\widehat{Y}$ and orbits in the coset space $G / H$ for the action of $\pi_{1}(Y)$. The fundamental group of the component corresponding to the orbit of the coset $g H$ is a conjugate of $i^{-1}\left(\mathrm{gHg}^{-1}\right)$ in $\pi_{1}(Y)$. 
Proof. Let $\widetilde{X}$ denote the universal cover of $X$, and let $\widetilde{Y}$ denote the subspace corresponding to $Y$. Pick $x_{o} \in \widetilde{X}$ a lift of $y_{0}$. Each component of $\widetilde{Y}$ contains some $g . x_{0}$. A loop $\gamma$ in $Y$ based at $y_{0}$ lifts to a path from $g . x_{0}$ to $g^{\prime} \cdot x_{0}$, for $g^{\prime}=i([\gamma]) . g$, where $[\gamma]$ denotes the element of $\pi_{1}(Y)$ represented by the loop $\gamma$. The points $g . x_{0}$ and $g^{\prime} \cdot x_{0}$ map to the same point of $\widehat{X}$ if and only if $g H=g^{\prime} H$. Hence there is a path in $\widehat{Y}$ from the image of $g \cdot x_{0}$ to the image of $g^{\prime} \cdot x_{0}$ if and only if the cosets $g H$ and $g^{\prime} H$ are in the same $\pi_{1}(Y)$-orbit, as claimed.

Each component of $\widehat{Y}$ is a covering space of $Y$, and so once we have chosen a basepoint we may identify its fundamental group with a subgroup of $\pi_{1}(Y)$. Taking different basepoints changes this subgroup by conjugation. The image of the point $g . x_{0}$ in $\widehat{Y}$ depends only on the coset $g H$. If we take as basepoint for a component of $\widehat{Y}$ the image of $g \cdot x_{0}$, then a loop $\gamma$ in $Y$ lifts to a loop in $\widehat{Y}$ based at $g . x_{0}$ if and only if the cosets $g H$ and $i([\gamma]) g H$ are equal, or equivalently if and only if $i([\gamma]) \in g \mathrm{Hg}^{-1}$.

Corollary 5 Suppose that a group $G$ is expressed as a free product with amalgamation, $G=H *_{L} K$, and that $\phi: G \rightarrow Q$ is such that $\phi: L \rightarrow Q$ is surjective. Then $\operatorname{ker}(\phi)$ is equal to the free product with amalgamation $H^{\prime} *_{L^{\prime}} K^{\prime}$, where $H^{\prime}=\operatorname{ker}(\phi) \cap H, L^{\prime}=\operatorname{ker}(\phi) \cap L$ and $K^{\prime}=\operatorname{ker}(\phi) \cap K$.

Proof. A model for the classifying space $B G$ can be made by joining copies of $B H$ and $B K$ by a cylinder $B L \times I$, where $I$ denotes the unit interval. Take this space to be the space $X$ in Lemma 4 , and for $\widehat{X}$ take the regular cover with fundamental group $\operatorname{ker}(\phi)$, so that $\widehat{X}$ is the classifying space for $\operatorname{ker}(\phi)$. Lemma 4 can be applied in the cases $Y=B H, Y=B K$ and $Y=B L \times I$. In each case, it follows that $\widehat{Y}$ is connected, and the fundamental group of $\widehat{Y}$ is $H^{\prime}, K^{\prime}$ or $L^{\prime}$ respectively. Hence $\widehat{X}$ is built by joining a copy of $B H^{\prime}$ and a copy of $B K^{\prime}$ via a cylinder $B L^{\prime} \times I$, and so $\operatorname{ker}(\phi) \cong H^{\prime} *_{L^{\prime}} K^{\prime}$.

Corollary 6 Suppose that $G=H * K$, and define a homomorphism $\phi: G \rightarrow$ $K$ as the identity homomorphism on $K$ and the trivial map on $H$. The kernel of $\phi$ is isomorphic to a free product of copies of $H$ indexed by the elements of $K$.

Proof. Build a classifying space $B G$ as the one-point union $B H \vee B K$, and apply Lemma 4 with $\widehat{X}$ being the regular cover corresponding to $\operatorname{ker}(\phi)$. In the case when $Y=B K$, we see that $\widehat{Y}$ is the universal covering space $E K$ 
of $B K$, and in the case when $Y=B H$, we see that $\widehat{Y}$ is a disjoint union of copies of $B H$ indexed by the elements of $K$. Hence $B(\operatorname{ker}(\phi))$ can be constructed by attaching copies of $B H$ indexed by the elements of $K$ to the contractible space $E K$.

Apart from the assertion concerning finite presentablility, the following theorem is a special case of [3, prop. 2.13(a)].

Theorem 7 (R. Bieri) Let $G=H *_{L} K$ be a free product with amalgamation, and suppose that both $H$ and $K$ are type $F$. Then for any $n \geq 1, G$ is $F P_{n}$ if and only if $L$ is $F P_{n-1}$. If $L$ is finitely generated, then $G$ is finitely presentable.

Proof. The assertions concerning the $F P_{n}$ conditions are a special case of $[3$, prop. 2.13(a)]. By hypothesis, there are finite models for $B H$ and for $B K$. If there is a model for $B L$ with finite 1-skeleton, then by gluing $B H, B L \times I$ and $B K$, one may construct a model for $B G$ with finite 2-skeleton. Hence in this case $G$ admits a finite presentation.

The group $H$ below was introduced by Higman, who proved that $H$ has the 'group theoretic' properties given in the following theorem [7]. The proof that $H$ has the stated 'homological properties' was given by Baumslag, Dyer and Heller in [1], where the group $H$ played an important role in their strengthened version of the Kan-Thurston theorem.

Theorem 8 (G. Higman, G. Baumslag, E. Dyer, A. Heller) Let $H$ be the group defined by the presentation

$$
H=\left\langle a, b, c, d: a^{b}=a^{2}, b^{c}=b^{2}, c^{d}=c^{2}, d^{a}=d^{2}\right\rangle .
$$

Then $H$ is an infinite torsion-free group, the presentation 2-complex for the above presentation is a classifying space for $H$, and $H$ admits no non-trivial quotient in which the images of the generators have finite order.

Corollary $9 H$ as above is a non-trivial torsion-free acyclic group with no proper finite-index subgroups. 


\section{The groups}

Lemma 10 Let $M$ be a module for Higman's group $H$ whose underlying abelian group is finitely generated. Then $H$ acts trivially on $M$.

Proof. Let $G=\operatorname{Aut}(M)$, the group of abelian group automorphisms of $M$. An $H$-module structure on $M$ is a homomomorphism $H \rightarrow G$. By Proposition $3, G$ is isomorphic to a subgroup of $G L_{n}(\mathbb{Z})$ for some $n$. Thus it suffices to show that there are no non-trivial homomorphisms $\phi: H \rightarrow G L_{n}(\mathbb{Z})$. For each $m>1$, let $\pi_{m}: G L_{n}(\mathbb{Z}) \rightarrow G L_{n}(\mathbb{Z} / m \mathbb{Z})$ denote the homomorphism 'reduction modulo $m$ '. By Corollary $9, H$ has no proper finite-index subgroups, and so the homomorphism

$$
\pi_{m} \circ \phi: H \rightarrow G L_{n}(\mathbb{Z} / m \mathbb{Z})
$$

must be trivial for each $m>1$. However, the only matrix in the kernel of all of the $\pi_{m}$ is the identity matrix, and so $\phi$ must be the trivial homomorphism.

Remark 11 For any group $G$, and any right $G$-module $A$, a left $G$-action on $A$ may be defined by $g * a=a g^{-1}$. This gives a bijection between the left and right $G$-module structures on any fixed abelian group $A$.

Proposition 12 Let $G_{0}$ be an infinite free product of copies of Higman's group $H$, and let $M$ be a right $G_{0}$-module whose underlying abelian group is finitely generated. Then $G_{0}$ acts trivially on $M$, and

$$
H_{0}\left(G_{0} ; M\right) \cong M, \quad H_{i}\left(G_{0} ; M\right)=0 \quad \text { for } i>0 .
$$

Proof. Let $M$ be as in the statement. By Lemma 10 and the remark following it, each copy of $H$ inside $G_{0}$ must act trivially on $M$. It follows that $G_{0}$ acts trivially on $M$. Since $H$ is acyclic, it follows that $G_{0}$ is also acyclic, and so the homology of $G_{0}$ with integer coefficients is isomorphic to the integral homology of the trivial group. The universal coefficient theorem allows one to compute the homology of $G_{0}$ with coefficients in any trivial module. Since each $H_{i}\left(G_{0} ; \mathbb{Z}\right)$ is free, the tor-term in the universal coefficient theorem vanishes, and so $H_{i}\left(G_{0} ; M\right) \cong M \otimes_{\mathbb{Z}} H_{i}\left(G_{0} ; \mathbb{Z}\right)$ for all $i$, giving the result claimed above. 
Corollary 13 The group $G_{0}$ as described above is FHT, is $F_{0}$ and is not $F P_{1}$.

Proof. There is a 2-dimensional $B G_{0}$ (consisting of the one point union of infinitely many copies of a 2-dimensional $B H)$, so $G_{0}$ has cohomological dimension at most 2. (In fact, since $G_{0}$ is not free its cohomological dimension is exactly 2, but we do not need this fact.) By Proposition 12, the homology groups $H_{i}\left(G_{0} ; M\right)$ are all finitely generated whenever $M$ is a right $G_{0}$-module whose underlying abelian group is finitely generated. A group is $F P_{1}$ if and only if it is finitely generated, and so $G_{0}$ is not $F P_{1}$. Every group is $F_{0}$.

To construct the rest of our examples, we will start by embedding $G_{0}$ in a group of type $F$. Let $J_{0}$ be the free product $H * \mathbb{Z}$, and define $\phi_{0}: J_{0} \rightarrow \mathbb{Z}$ by the identity map on $\mathbb{Z}$ and the trivial map from $H$ to $\mathbb{Z}$. Applying Corollary 6 , we see that $\operatorname{ker}\left(\phi_{0}\right)$ is isomorphic to a free product of infinitely many copies of the Higman group $H$. From now on, we shall identify $G_{0}$ with $\operatorname{ker}\left(\phi_{0}\right) \leq J_{0}$.

Let $\mathbb{F}_{2}$ denote the free group on two generators, and let $\psi: \mathbb{F}_{2} \rightarrow \mathbb{Z}$ be the homomorphism that sends each of the two generators to $1 \in \mathbb{Z}$.

Now suppose that we have already defined a group $J_{n}$ and a homomorphism $\phi_{n}: J_{n} \rightarrow \mathbb{Z}$. Define a new group $J_{n+1}$ containing $J_{n}$ as a direct factor, and a new homomorphism $\phi_{n+1}: J_{n+1} \rightarrow \mathbb{Z}$ extending $\phi_{n}$ by

$$
J_{n+1}=J_{n} \times \mathbb{F}_{2}, \quad \phi_{n+1}(g, h)=\phi_{n}(g)+\psi(h) \quad \text { for all } g \in J_{n} \text { and } h \in \mathbb{F}_{2} \text {. }
$$

For each $n$, we shall identify $J_{n}$ with $J_{n} \times\{1\} \leq J_{n+1}$. For $n>0$, define $G_{n}=\operatorname{ker}\left(\phi_{n}: J_{n} \rightarrow \mathbb{Z}\right)$.

Proposition 14 For each $n \geq 0, G_{0}$ is a normal subgroup of $G_{n}$, and $G_{n} / G_{0} \cong\left(\mathbb{F}_{2}\right)^{n}$. For each $n \geq 0$, there is an isomorphism $G_{n+1} \cong J_{n} *_{G_{n}} J_{n}$.

Proof. $J_{0}$ is a direct factor of $J_{n}$, and $G_{0}$ is a normal subgroup of $J_{0}$. It follows that $G_{0}$ is normal in $J_{n}$ and that

$$
J_{n} / G_{0} \cong\left(J_{0} / G_{0}\right) \times \mathbb{F}_{2}^{n} \cong \mathbb{Z} \times \mathbb{F}_{2}^{n}
$$

$G_{0}$ is contained in $G_{n}=\operatorname{ker}\left(\phi_{n}\right)$, and so there is an induced homomorphism $\bar{\phi}_{n}: J_{n} / G_{0} \rightarrow \mathbb{Z}$. Under the above isomorphism $J_{n} / G_{0} \cong \mathbb{Z} \times \mathbb{F}_{2}{ }^{n}$, the homomorphism $\bar{\phi}_{n}$ corresponds to the homomorphism which sends $\left(r, s_{1}, \ldots, s_{n}\right) \in$ 
$\mathbb{Z} \times \mathbb{F}_{2}{ }^{n}$ to $r+\psi\left(s_{1}\right)+\cdots+\psi\left(s_{n}\right)$. Since this map restricts to $\mathbb{Z} \times\{e\}^{n} \leq \mathbb{Z} \times \mathbb{F}_{2}{ }^{n}$ as the identity map of $\mathbb{Z}$, it follows that $\operatorname{ker}\left(\bar{\phi}_{n}\right)$ is isomorphic to $\mathbb{F}_{2}{ }^{n}$. Hence

$$
G_{n} / G_{0}=\operatorname{ker}\left(\bar{\phi}_{n}\right) \cong \mathbb{F}_{2}^{n}
$$

as claimed.

We may write $\mathbb{F}_{2}=\mathbb{Z} * \mathbb{Z}$, and thus we may write

$$
J_{n+1}=J_{n} \times(\mathbb{Z} * \mathbb{Z})=\left(J_{n} \times \mathbb{Z}\right) *_{J_{n}}\left(J_{n} \times \mathbb{Z}\right) .
$$

Let $\phi^{\prime}$ be the restriction of $\phi_{n+1}$ to one of the two copies of $J_{n} \times \mathbb{Z}$. The map $\phi^{\prime}$ is given by the formula $\phi^{\prime}(g, r)=\phi_{n}(g)+r$. In particular, the restriction of $\phi^{\prime}$ to the $\mathbb{Z}$ direct factor is the identity, and it follows that $\operatorname{ker}\left(\phi^{\prime}\right)$ is isomorphic to $J_{n}$. The isomorphism between $G_{n+1}$ and $J_{n} *_{G_{n}} J_{n}$ follows by applying Corollary 5 to $\phi_{n+1}$.

Theorem 15 For each $n \geq 0$, the group $G_{n}$ is torsion-free, is FHT and is $F_{n}$, but is not $F P_{n+1}$.

Proof. The group $J_{n}$ has a finite $(n+2)$-dimensional classifying space, so $J_{n}$ is type $F$. Also $G_{n}$ (as a subgroup of $J_{n}$ ) must have finite cohomological dimension, and so must be torsion-free. (In fact the cohomological dimensions of $J_{n}$ and $G_{n}$ are both equal to $n+2$.) The group $G_{0}$ is $F P_{0}$ but not $F P_{1}$. The assertion that $G_{n}$ is $F P_{n}$ but not $F P_{n+1}$ follows by induction, using Bieri's theorem (Theorem 7) and the description $G_{n+1} \cong J_{n} *_{G_{n}} J_{n}$. It follows that $G_{1}$ is type $F_{1}$. The assertion that $G_{n}$ is finitely presented for $n \geq 2$ also follows from Theorem 7 . In the case when $n \geq 2$, since $G_{n}$ is finitely presented and is $F P_{n}$, it follows that $G_{n}$ is type $F_{n}$.

It remains to check that whenever $M$ is a right $G_{n}$-module whose underlying abelian group is finitely generated, then each $H_{i}\left(G_{n} ; M\right)$ is finitely generated. For this we use the Lyndon-Hochschild-Serre (or LHS) spectral sequence for the group extension $G_{0} \rightarrow G_{n} \rightarrow G_{n} / G_{0}$. Let $M$ be a right $G_{n^{-}}$ module whose underlying abelian group is finitely generated. The $E^{2}$-page of the LHS-spectral sequence has

$$
E_{i, j}^{2}=H_{i}\left(G_{n} / G_{0} ; H_{j}\left(G_{0} ; M\right)\right) .
$$

By Proposition 12, the subgroup $G_{0}$ acts trivially on $M$, and $H_{0}\left(G_{0} ; M\right)=$ $M, H_{j}\left(G_{0} ; M\right)=0$ for $j>0$. Also $G_{n} / G_{0} \cong \mathbb{F}_{2}^{n}$ is a group of type $F$. 
Since the spectral sequence has $E_{i, j}^{2}=0$ for $j \neq 0$, it must collapse, giving isomorphisms

$$
H_{i}\left(G_{n} ; M\right) \cong E_{i, 0}^{2} \cong H_{i}\left(G_{n} / G_{0} ; M\right) \cong H_{i}\left(\mathbb{F}_{2}{ }^{n} ; M\right)
$$

Since $\mathbb{F}_{2}{ }^{n}$ is of type $F$, it follows that each $H_{i}\left(G_{n} ; M\right)$ is finitely generated as claimed.

\section{References}

[1] G. Baumslag, E. Dyer and A. Heller, The topology of discrete groups, J. Pure Appl. Algebra 16 (1980) 1-47.

[2] M. Bestvina and N. Brady, Morse theory and finiteness properties of groups, Invent. Math. 129 (1997) 445-470.

[3] R. Bieri, Homological Dimension of Discrete Groups, Queen Mary College Mathematics Notes, Queen Mary College, University of London, (1976).

[4] K. S. Brown, Euler characteristics of discrete groups and $G$-spaces, Invent. Math. 27 (1974) 229-264.

[5] K. S. Brown, Euler characteristics of groups: the $p$-fractional part, Invent. Math. 29 (1975), 1-5.

[6] K. S. Brown, Cohomology of Groups, Graduate Texts in Mathematics 87 Springer Verlag (1982).

[7] G. Higman, A finitely generated infinite simple group, J. London Math. Soc. 24 (1951) 61-64.

[8] M. Saadetoğlu, Finiteness conditions and Bestvina-Brady groups, PhD Thesis, School of Mathematics, University of Southampton (2005).

[9] J.-P. Serre, Cohomologie des groupes discrets, Prospects in Mathematics, 77-169, Ann. of Math. Studies 70 (1971) Princeton Univ. Press. 


\section{Authors' addresses:}

Ian Leary: Department of Mathematics, The Ohio State University, 231 West 18th Avenue, Columbus, Ohio 43210-1174, United States.

and

School of Mathematics, University of Southampton, Southampton, SO17

1BJ, United Kingdom.

leary@math. ohio-state.edu

Müge Saadetoğlu: School of Mathematics, University of Southampton,

Southampton, SO17 1BJ, United Kingdom.

and

Department of Mathematics, Eastern Mediterranean University,

Gazimağusa, Turkish Republic of Northern Cyprus.

muge. saadetoglu@emu.edu.tr 